Sara Lanna, Mesomede. Inno a Фúøı. Introduzione, testo critico, traduzione e commento

"Quaderni dei Seminari Romani di Cultura Greca", 15, Roma, Edizioni Quasar, 2013, pp. 275, ISBN 9788871405148, € 31,00

\title{
Antonietta Provenza
}

\section{(2) OpenEdition}

12 Journals

Edizione digitale

URL: http://journals.openedition.org/mythos/673

DOI: $10.4000 /$ mythos. 673

ISSN: 2037-7746

Editore

Salvatore Sciascia Editore

\section{Edizione cartacea}

Data di pubblicazione: 1 dicembre 2017

Paginazione: 171-175

ISSN: 1972-2516

\section{Notizia bibliografica digitale}

Antonietta Provenza, «Sara Lanna, Mesomede. Inno a Фúøıç. Introduzione, testo critico, traduzione e commento », Mythos [Online], 11 | 2017, online dal 24 septembre 2019, consultato il 25 septembre 2020. URL : http://journals.openedition.org/mythos/673; DOI : https://doi.org/10.4000/mythos.673 


\section{Sara Lanna}

Mesomede. Inno a Фúøıc. Introduzione, testo critico, traduzione e commento

("Quaderni dei Seminari Romani di Cultura Greca", 15), Roma, Edizioni Quasar, 2013, pp. 275, ISBN 9788871405148, € 31,00.

\section{Antonietta Provenza - Università degli Studi di Palermo - antonietta.provenza@unipa.it}

II citarodo cretese Mesomede, l'unico che si conosca per l'età imperiale, fu attivo nella prima metà del II sec. d.C. Liberto ed amico dell'imperatore Adriano (Suid. $\mu$ 668), fu musico di corte e autore di vó $\mu$ o

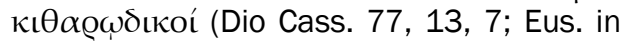
Hieronym. Chron. 284 F. 22-24, p. 202 Helm). Sono pervenuti tredici componimenti a lui attribuiti (l'edizione di riferimento è $E$. Heitsch, Die griechischen Dichterfragmente der römischen Kaiserzeit, I, Göttingen $\left.1963^{2}, 24-32\right)$, alcuni con notazione musicale (cf. E. Pöhlmann - M. West, Documents of Ancient Greek Music, Oxford 2001, 92115), che dovevano essere eseguiti ancora nel IV sec.: Sinesio cita alcuni versi dell'Inno A Nemesi, che, dice, "cantiamo con l'accompagnamento della lira” (Ep. 95). Di tali com-

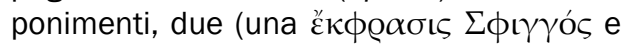
un carme in cui si descrive la lavorazione del vetro) si leggono nell'Anthologia Graeca (A.P. XIV, 63 = Mesom. 12 Heitsch; A. PI. 323 = Mesom. 13 Heitsch); altri tre (I'invocazione Alla Musa, gli Inni A Helios e A Nemesi), con notazione musicale, in vari manoscritti; otto componimenti furono scoperti da Konstantin Horna nel 1903 presso la Biblioteca Vaticana in un codice miscellaneo, l'Ottoboniano graecus 59, che rimane codex unicus per i carmina nrr. 4-11 (gli Inni A Physis, A

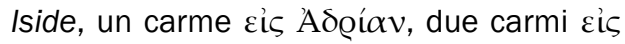

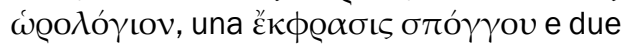

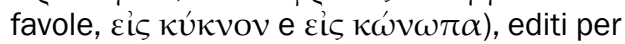
la prima volta dallo stesso Horna solo nel 1928 (Die Hymnen des Mesomedes, "Sb. Wien, Phil.-hist. Klasse”, 207, Vienna - Leipzig 1928).

Sara Lanna fa precedere questa sua pregevole edizione critica con traduzione e commento dell'Inno a Physis da una ricca introduzione (5-89), che illustra la biografia
(5-7), il contesto storico-culturale di Mesomede (16-20), il suo stile (7-15), la tradizione manoscritta (33-67), lo stato dell'arte e il rapporto dell'innografia mesomedea, e dell'Inno in questione, col genere (67-89). Seguono il testo critico con la traduzione in italiano e la nota critica (91-94), e un esaustivo commento (95-222), strutturato secondo lemmi o gruppi di lemmi ed arricchito dal riferimento ad una vastissima letteratura, in particolare orfica, neoplatonica e cristiana. II commento raggiunge l'obiettivo di spiegare puntualmente il testo dell'Inno, evidenziandone in una fine esegesi le peculiarità lessicali e concettuali sia rispetto al genere, sia - più ampiamente - in rapporto alla poesia greca fino agli autori cristiani.

Il volume è corredato da due appendici, la prima (223-229) comprendente le Tavole dei lemmi, che illustrano verso per verso in successivi schemi, rispettivamente, il rapporto del lessico dell'Inno $A$ Physis con la tradizione ("epiteti tradizionali", "espressioni tradizionali", "epicismi"), i suoi modelli ("modelli lirici", "modelli tragici", "modelli alessandrini", "modelli prosastici"), e i suoi legami con la tradizione e con la filosofia e il misticismo ("concetti filosofici", "espressioni interpretabili in senso orfico/misteriosofico", "confronto con Inni orfici", "confronto con Lamine orfiche", "espressioni del $\mathrm{Ne}$ opitagorismo-Medioplatonismo (confronto con Oracoli caldaici e Proclo)"). Segue uno schema relativo alle innovazioni linguistiche peculiari dell'Inno ("prima attestazione" nella letteratura e nella religione greca, "sintagmi inediti", "hapax"), uno che restituisce le tracce lessicali del sentire religioso di Mesomede ("espressioni indicanti mutato sentimento religioso") - a cui si connettono i "termini con significato sviluppatosi in età 
imperiale (in letteratura)" -, e inoltre uno schema riguardante gli elementi di novità relativi al rapporto con la divinità, che si esprimono in "prerogative nuove" e, a livello stilistico e sintattico, nelle "novità strutturali", nei "connettivi funzionali" e nelle "figure retoriche".

La seconda appendice (230-238) comprende i carmi nrr. 1-3 e 5-13 Heitsch; il testo è rivisto in più punti, e l'apparato critico è integrato.

Riguardo all'origine della tradizione di Mesomede, presumibilmente utilizzato nel Medioevo come specimen sul quale impostare l'apprendimento della cetra (cf. M. West, Ancient Greek Music, Oxford 1992, 50), S.L. (39) riprende l'ipotesi di Pöhlmann - West $(2001,115)$, secondo cui essa risiederebbe in uno dei trattati di teoria musicale dai quali venne estratta la Hormasia (il tito-

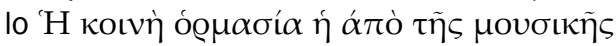
$\mu \varepsilon \tau \alpha \beta \lambda \eta \theta \varepsilon \tilde{\imath} \sigma \alpha$ designa un insieme di tavole sull'antica notazione musicale greca contenute in excerpta di teoria musicale editi in Th. Reinach, "Fragments musicologiques inédits", Révue des Études Grecques 10 (1897), 313-327).

Grande attenzione è dedicata nell'introduzione alle caratteristiche della poetica di Mesomede, al metro (21-26) e a quanto si può desumere della musica (26-33) dei suoi carmi. II citarodo cretese trova il suo modello musicale nella tradizione arcaica, individuando nel genere diatonico e nel ritmo anapestico le forme più adatte alla solennità richiesta all'inno. In particolare, egli si avvale nel preludio dell'Inno A Helios e nell'Inno $A$ Physis di un verso eptasillabo costituito da tutte sillabe lunghe, interpretato come paremiaco con frequente presenza di fine di parola dopo la quinta sillaba (di fatto, un paremiaco in cui tutti gli anapesti sono sostituiti da spondei; ogni verso dell'Inno $A$ Physis, tranne i vv. 7 e 15, è costituito esclusivamente da sillabe lunghe). Modo prevalente nei componimenti mesomedei è il lidio, o l'ipolidio (n. 175: il lidio diatonico è il modo abituale della citarodia e, per la sua semplicità, è il modo di riferimento nei trattati di musica), e si riscontrano uno scarso uso del melisma e poche (talvolta nessuna) modulazioni di tonos, in un periodo (II-IV sec.) in cui lo stile melismatico era molto diffuso a Roma. II tempo dilatato e la statica solennità dell'andamento melodico proiettavano il fedele nella dimensione ieratica del rito, che rappresentava una rottura rispetto alla quotidianità.

Gli Inni di Mesomede rappresentano un modello metrico e ritmico nel passaggio "dall'antico culto greco all'innografia cristiana" (26), che riprende i moduli anapestici della poesia adrianea. La studiosa riscontra a più riprese come Mesomede costituisca un modello per Sinesio (cf. al riguardo anche S. Lanna, "Sinesio e Mesomede: continuità di ritmi, significanti e significati tra religiosità orfico-pagana e neoplatonico-cristiana", Seminari Romani di Cultura Greca, 12 (2009), 95-113) e, sulla scorta di Gevaert (F. A. Gevaert, La mélopée antique dans le chant de l'église latine. Suit et complément de l'histoire et théorie de la musique de l'antiquité, Gand 1895, 62-82), evidenzia che sull'innografia cristiana si riscontra l'influsso della citarodia greco-romana, aggiungendo un utile riferimento al POxy. 1786 (III-IV sec.), contenente il più antico pezzo di poesia della chiesa cristiana, con notazione musicale, riconducibile agli anapesti di età adrianea.

Mesomede trova nel dialetto dorico il legame più appropriato con la lirica corale e, al tempo stesso, il riferimento più colto alla lingua di Pitagora, dal momento che i suoi Inni si inseriscono nella corrente religiosa orfico-neopitagorica, i cui prodotti letterari sono in dialetto dorico. Sul piano stilistico e lessicale, S. L. lascia emergere la complessa natura dell'aemulatio del citarodo nei confronti della tradizione poetica ellenistica: Mesomede appare sensibile, da un lato, all'accumulo lessicale proprio degli Inni Orfici, ed incline, dall'altro, ad una ricercata raffinatezza stilistica che rimane Iontana dall'artificiosità; dal punto di vista del lessico e dei sintagmi e delle immagini, sebbene non attinga "di frequente ai lirici greci, non li evita" (8-9 n. 26). 
Riguardo alla performance e all'occasione degli Inni, la studiosa (81-82) si accosta alle osservazioni di Whitmarsh (cf. T. Whitmarsh, "The Cretan Lyre Paradox: Mesomedes, Hadrian and the Poetics of Patronage", in B. E. Borg (ed.), Paideia: The World of Second Sophistic, Berlin - New York 2004, 377-402, in part. 383), secondo cui non si può escludere che questi fossero composti per reali occasioni religiose rituali; tale tesi si contrappone alle interpretazioni di quanti, a partire da Wilamowitz (cf. U. von WilamowitzMoellendorff, Griechische Verskunst, Berlin 1921, 606, secondo cui solo I'Inno A Iside era destinato al culto), considerano gli Inni di Mesomede come testi non pensati per il culto (essi non sono inclusi in W. D. Furley - J. M. Bremer, Greek Hymns, I-II, Tübingen 2001, I, 47). L'Inno A Physis, pervenutoci senza la melodia, è ritenuto dalla studiosa "esempio di un inno ormai svincolato dagli elementi che lo collegano alla tradizione e divenuto espressione di un canto personale alla divinità, o legato alle esigenze di un rito ristretto a pochi", per cui si può escludere "la presenza di un coro, anche muto" (29).

Con un'attenta indagine dei presupposti storico-religiosi dell'Inno in questione in particolare dell'ambito orfico-pitagorico (201: I'Inno A Physis rappresenta, insieme con le lamine orfiche, una testimonianza della continuità della dottrina escatologica orfica), adombrato già nella didascalia

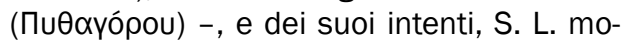
stra come esso delinei per il fedele un percorso di salvezza che si avvale della ricerca costante della rettitudine e di una parola conforme alla verità divina, veicolo di salvezza sia per il corpo, sia per l'anima. Di questo percorso verso la salvezza, la poesia di Mesomede intende porsi come tramite: il poeta, che si definisce al v. $20 \beta \alpha \kappa \chi \varepsilon v \tau \alpha ́$ s (forma dorica; il termine, costruito sul tema di $\beta \alpha \kappa \chi \varepsilon v ́ s$, è attestato per i fedeli di Dioniso solo in quest'Inno e nell'Etymologicum Magnum [ 10 Lasserre-Livadaras]; cf. 197), è egli stesso "iniziato", e si pone come guida verso la verità e la salvezza divina comunicando una "бoфí $\alpha$ di vita" (150).
II nostro Inno appare per molti aspetti peculiare: Physis non costituisce una divinità tradizionale, né la divinizzazione di un principio, ma è "la stessa natura innalzata al rango di divinità assoluta" (85). I primi versi si distinguono rispetto alla convenzione del genere contravvenendo al principio della correttezza rituale del nome del dio invocato, che costituiva una premessa indispensabile per propiziarsene la benevolenza: Physis è detta tale solo nel titolo, mentre al v. 3 è invocata come "Notte" (Núk), "Luce"

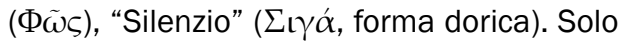
al v. 6 , e in nessun'altra parte dell'Inno, essa è invocata col nome, Rea, che identifica la Grande Madre dei Misteri (significativa è la disamina del vocativo $\mu \tilde{\alpha} \tau \varepsilon \varrho$ a 104-105). S.L. si addentra in un'ampia indagine sulla

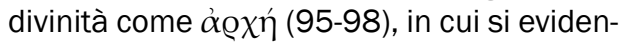
zia come la scelta di Mesomede di iniziare il carme proprio con dò $\alpha \dot{\alpha}$ in posizione enfatica sia "senza paralleli in un inno cultuale" (97). Di fatto, “l'intera iunctura del v. 1,

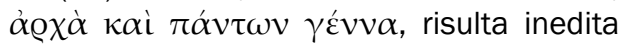
sia a livello strutturale, sia dal punto di vista semantico" (101), poiché la divinità è esaltata, al tempo stesso, in chiave metafisica e genetica come origine di tutte le cose. II sincretismo con la religione orfica è adombrato sia dall'appellativo 'Pcín, sia dalla connessione tra Núk e $\Phi \tilde{\omega} s:$ interessanti sono il riferimento a HOrphNoct. 3.1, in cui la Notte è legata all'aspetto generativo dell'amore ed è

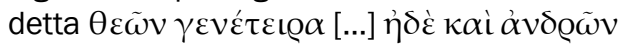
(111), e l'accostamento di Physis con Iside, anch'essa madre, e legata sia alla notte, sia alla luce (112-113). Nuovo è anche il pre-

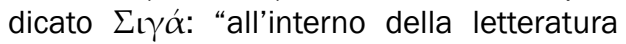
greca non esiste una personificazione o deificazione del silenzio prima di Mesomede" (119); anch'esso rinvia all'ambito dei Misteri, come S.L. illustra in puntuali riferimenti letterari orfici e neoplatonici, ed epigrafici.

Tra le scelte testuali, emerge la concordanza della studiosa con Horna $(1928,10$; la congettura è accolta in seguito in Heitsch 1963, 27), che al v. 4 emenda il tràdito $\mu \tilde{v} \theta \varepsilon$ (corruttela dell'Ottoboniano greco 59) in $\mu v ́ \sigma \tau \alpha \varsigma$. Questo termine è "pregnante 
per il nostro inno" dal punto di vista dell'etimologia ( $\mu v ́ \sigma \tau \eta \varsigma$ da $\mu v ́ \omega$, "tenere la bocca chiusa"), data l'invocazione della Natura al v. 3 come $\Sigma$ l $\alpha$ ' (124). Il testo così emendato prevede che la Natura "custodisca" (è usato il verbo ф@ov@é $\omega)$ gli iniziati al suo culto (121-126).

Ancora in chiave genetica è presentata la "divinizzazione" degli iniziati: la Natura

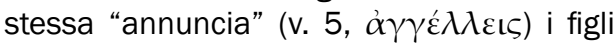

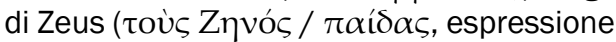
per la quale non esiste la possibilità di raffronti puntuali), ovvero afferma per i $\mu v ́ \sigma \tau \alpha$ เ "una filiazione che vanta lo stesso Zeus come genitore divino" (128). Chiara e condivisibile è l'interpretazione fornita da S. L. di questi difficili versi, senza paralleli puntuali in tutta la letteratura greca: liberi dal ciclo della metensomatosi, gli iniziati sono puri, divenuti dèi da mortali che erano (130). L'ambito misterico è evocato anche dallo

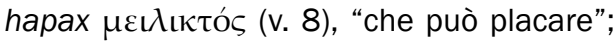
all'interno di una vasta disamina sulla presenza del verbo $\mu \varepsilon \iota \lambda i ́ \sigma \sigma \omega$ nella letteratura orfica (138-142), S. L. non include tuttavia

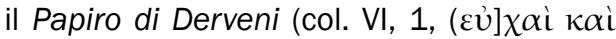

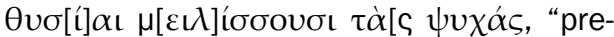
ghiere e sacrifici addolciscono le anime").

A partire dal v. 9, il poeta dà voce al suo "io", ovvero ai suoi desideri rispetto all'ambito di azione di Physis. Unica è la definizione dell'itinerario dell'anima, rappresentato al

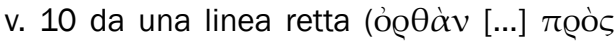
$\gamma \varrho \alpha \mu \mu \alpha ́ \alpha)$, ovvero da un percorso verso una meta (146-148). Dal v. 15 l'invocazione riguarda il Sole, “immagine visibile dell'essenza della Natura" (162), fonte di beatitudine per l'iniziato, che nella sua luce si protende verso l'eternità, pur nella transitorietà del suo misero corpo, in ragione del quale il poeta chiede all'astro - invocato come Ai $\omega$ v (v. 17, ampiamente discusso a 172-182), in opposizione alla caducità della propria dimensione umana - di avere pietà di lui (182: concordando con Whitmarsh 2004, S. L. riscontra in questi versi tracce di una "poetica di patronato"). Anche questo elemento appare estrinseco rispetto alla tradizione innografica, che non prevede una richiesta di compassione da parte del fedele (212).

Interessante e convincente è l'interpretazione dei versi finali, in cui la "miseria" della condizione umana è connotata dall'aggettivo $\delta \varepsilon \iota \lambda o ́ s$, che definisce il contrasto tra la materia del corpo e l'anima che tende al divino, e il disagio derivante da tale contrasto (217-222). Nell'ultima sezione dell'Inno, il poeta prega per sé, "e nella sua voce poteva rispecchiarsi solo chi era parte della stessa cerchia religiosa di orientamento "mistico"" (88).

A Helios, invocato come luce che manifesta Physis, il poeta chiede di "versare" su di lui (il verbo usato è $\chi \dot{\varepsilon} \omega$, in senso religioso-rituale; 195-197), iniziato ai Misteri, “una san-

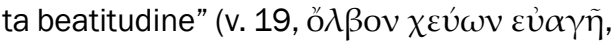
sintagma del tutto inedito; cf. 196-197). Riguardo a ǒ $\lambda \beta o \varsigma$, la "felicità" più completa ed elevata, dono degli dèi, S.L. compie una ricca e complessa disamina (187-193): si conclude che l'aspetto spirituale del termine avrebbe la sua prima attestazione proprio

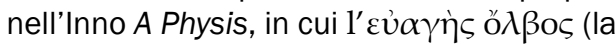
felicità che deriva dalla completa sottomissione del fedele alla volontà del dio, come indica l'aggettivo, esaminato a 193-195) giunge al poeta iniziato direttamente dalla luce che proviene dagli occhi di Helios.

Esaustiva e utile, anche per uno studio dei testi orfici e della complessa problematica dell'identificazione del miste col dio

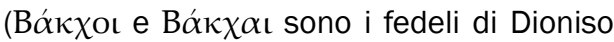
nel corso dei riti misterici), è la disamina di $\beta \alpha \kappa \chi \varepsilon v \tau \eta ́ s ~(197-201$, mai attestato prima di Mesomede in riferimento al fedele, ma solo al dio, a differenza di $\beta \alpha ́ \alpha \chi o \varsigma)$, segno di "un forte anelito di raggiungere la divinità, di "indiarsi"” (201). Il poeta-baccheuta invo-

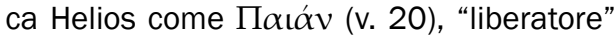
dell'anima del fedele dai vincoli del corpo affinché ascenda pura e conforme alla divinità. L'appellativo (202-204), che evidenzia una identificazione tra Helios e Dioniso peraltro operante in ambito orfico, e qualifica in età arcaica una divinità indipendente, come opportunamente si rileva, è in seguito associato con Apollo, Asclepio (202: già in Aristoph. Pl. 636) - figlio di Apollo - con 
Dioniso e, appunto, con Helios. S. L. rileva che il peana di Filodemo a Dioniso (nr. 39 in L. Käppel, Paian. Studien zur Geschichte einer Gattung, Berlin 1992; 2. 5 Furley Bremer 2001; databile al 340-339 a.C., per le festività in occasione dell'inaugurazione del sesto tempio di Delfi) costituirebbe la prima attestazione dell'invocazione rituale di Dioniso come Peana; si può tuttavia aggiungere che non è possibile escludere che ciò avvenisse anche prima, come mostra ad es. Eur. fr. 477 Kannicht (dalla perduta tragedia Licimnio), in cui tra Apollo e Dioniso opera un sincretismo che associa gli epiteti

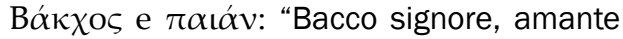
dell'alloro, Apollo dalla lira melodiosa, pea-

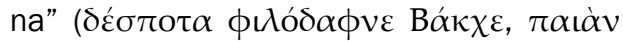

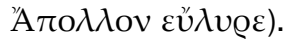

Una ricchissima bibliografia conclude lo studio; ad essa avrebbe potuto aggiungersi l'esaustivo volume di T. Power (The Culture of Kitharôidia, Cambridge (Mass.) - London 2010).

Nell'accurata resa mi limito a segnalare un solo refuso: Talete di Gortina anziché Taleta (63).

Lo studio di S. L. giunge pertanto molto opportunamente a colmare una lacuna nell'ambito della conoscenza dell'innografia greca: il pagano Mesomede, testimone del revival dell'Orfismo e del Pitagorismo in età imperiale, ci appare come un tramite tra la poesia arcaica e classica e l'innografia cristiana. 